\title{
Proliferation of community exercise programs with limited evidence and expertise: safety implications.
}

Josefa Domingos ${ }^{1,2^{\star}}, \mathrm{MSc}$; John Dean ${ }^{3}, \mathrm{MA}$ CCC-SLP; Catarina Godinho ${ }^{4}$, PhD; Filipe Melo, $\mathrm{PhD}^{1}$

${ }^{1}$ Laboratory of Motor Behavior, Sport and Health Department, Faculty of Human Kinetics, University of Lisbon, Portugal,

2 Department of Neurology, Donders Institute for Brain, Cognition and Behaviour, Radboud University Medical Center, Nijmegen, The Netherlands;

3 The Triad Health AI, USA;

${ }^{4}$ Centro de Investigação Interdisciplinar Egas Moniz (CiiEM),

Instituto Universitário Egas Moniz, Portugal.

*Corresponding Author: Josefa Domingos

Address: Laboratory of Motor Behavior, Sport and Health Department, Faculty of Human Kinetics, University of Lisbon, Portugal. Estr. da Costa MB, 1495-687 Cruz

Quebrada

Email: domingosjosefa@gmail.com

Total words: 491

Running title: Proliferation of community exercise programs

Key words: Parkinson Disease, Exercise, Physiotherapy, expertise, safety issues

Financial Disclosure/Conflict of Interest concerning the research related to the manuscript: All relevant disclosures and conflict of interests are detailed/ described/ listed at the end of this article.

Funding sources for the study: none 
A number of research studies underscore the beneficial effects that physiotherapy and exercise can have on functional activities involving gait, transfers, and balance in Parkinson's disease (PD) (1). People with Parkinson's (PwP) can receive this care in a range of settings from various professionals. Evidence is emerging that supports a number of community programs for PwP that incorporate different non-conventional types of group exercises, including dance (2), boxing (3), Nordic walking (4), Tai Chi (5), and aquatic exercise (6). There are reasons to be optimistic that these new initiatives may be a long-term cost-effective and easily accessible care strategy for ongoing exercise for PwP. However, data regarding their true benefits and the best approaches to implementation are still limited. Notably, there is an optimal minimal level of diseasespecific expertise necessary, particularly with respect to safely integrating exercises and reducing the risk of falls and other possible safety issues $(1,7)$. By prematurely referring PwP to exercise approaches that do not have robust evidence and are led by instructors with a lack of professional expertise, health professionals may favor unnecessary procedures, foster unrealistic expectations in PwP (particularly for those with less favorable profiles), and could ultimately be putting patients at risk of falls and/or injury (Fig.1).

As such, several important questions regarding specificity to PD and safety issues are now arising that should fuel future research. First, should we obtain more evidence about these community exercise programs and then implement them within the community setting, or should we start these programs first, even if little evidence is available? Second, taking into account the financial considerations, accessibility and safety issues, and the progressive nature of the disease, who should implement such community programs? Should physiotherapists incorporate boxing, dance, or tai chi into to their clinical practice? Or would it be more feasible while equally effective to train exercise instructors who teach community programs?

Safe evidence-based practices must be a priority for care in all settings, including the community. Several courses of action may be needed to address the gap between expertise, evidence, its dissemination, and implementation into community exercise practices.

Programs must be made available and accessible to PwP by reducing critical financial and travel barriers. If evidence-based practices are not covered or are inaccessible, they will simply not be used. Even if PwP may have limited access to evidenced-based care, that care, when delivered, should be delivered by instructors with PD expertise. It is 
critical to enhance expertise among professionals who deliver these exercise programs through specific training, adequate ongoing educational support, and continuous contact with PwP (1).

Additionally, we also need to enhance public and PwP awareness of evidence-based exercise programs and their locations. Increasing public interest in community exercise programs may also potentially positively influence the direction of clinical research and advance clinical practice.

All these actions can begin to guide us away from care disparities and promote better care for PwP. Without them, no amount of evidence will matter.

Acknowledgment: not applicable

\section{Author roles}

(1) Research Project: A. Conception, B. Organization, C. Execution; (2) Statistical Analysis: A. Design, B. Execution, C. Review and Critique; (3) Manuscript: A. Writing of the First Draft, B. Review and Critique.

J.M.D.: 1A, 1B, 1C, 3A, 3B,

C.G.: $1 \mathrm{C}, 3 \mathrm{~A}, 3 \mathrm{~B}$,

J.D.: 1C, 3A, 3B

F.M.: 1A, 3B

Financial disclosures: none

\section{References}

1. Keus SHJ, Munneke M, Graziano M. European Physiotherapy Guideline for Parkinson's disease. The Netherlands. 2014.

2. Duncan RP, Earhart GM. Randomized controlled trial of community-based dancing to modify disease progression in Parkinson disease. Neurorehabil Neural Repair. 2012;26(2):132-43. 
3. Combs SA, Diehl MD, Chrzastowski C, Didrick N, McCoin B, Mox N, et al. Community-based group exercise for persons with Parkinson disease: a randomized controlled trial. NeuroRehabilitation. 2013;32(1):117-24.

4. Monteiro EP, Franzoni LT, Cubillos DM, de Oliveira Fagundes A, Carvalho AR, Oliveira HB, et al. Effects of Nordic walking training on functional parameters in Parkinson's disease: a randomized controlled clinical trial. Scand J Med Sci Sports. 2017;27(3):351-8.

5. Choi HJ. Effects of therapeutic Tai chi on functional fitness and activities of daily living in patients with Parkinson disease. J Exerc Rehabil. 2016;12(5):499-503.

6. Carroll LM, Volpe D, Morris ME, Saunders J, Clifford AM. Aquatic Exercise Therapy for People With Parkinson Disease: A Randomized Controlled Trial. Arch Phys Med Rehabil. 2017;98(4):631-8.

7. Aminoff MJ, Christine CW, Friedman JH, Chou KL, Lyons KE, Pahwa R, et al. Management of the hospitalized patient with Parkinson's disease: current state of the field and need for guidelines. Parkinsonism Relat Disord. 2011;17(3):139-45. 